\title{
ON THE RETURN TIME FOR A REFLECTED FRACTIONAL BROWNIAN MOTION PROCESS ON THE POSITIVE ORTHANT
}

\author{
CHIHOON LEE, ${ }^{*}$ Colorado State University
}

\begin{abstract}
We consider a $d$-dimensional reflected fractional Brownian motion (RFBM) process on the positive orthant $S=\mathbb{R}_{+}^{d}$, with drift $r^{0} \in \mathbb{R}^{d}$ and Hurst parameter $H \in\left(\frac{1}{2}, 1\right)$. Under a natural stability condition on the drift vector $r^{0}$ and reflection directions, we establish a return time result for the RFBM process $Z$; that is, for some $\delta, \kappa>0$, $\sup _{x \in B} \mathrm{E}_{x}\left[\tau_{B}(\delta)\right]<$ $\infty$, where $B=\{x \in S:|x| \leq \kappa\}$ and $\tau_{B}(\delta)=\inf \{t \geq \delta: Z(t) \in B\}$. Similar results are known for reflected processes driven by standard Brownian motions, and our result can be viewed as their FBM counterpart. Our motivation for this study is that RFBM appears as a limiting workload process for fluid queueing network models fed by a large number of heavy-tailed ON/OFF sources in heavy traffic.
\end{abstract}

Keywords: Reflected fractional Brownian motion; heavy traffic theory; return time

2010 Mathematics Subject Classification: Primary 60G22

Secondary 90B18; 60G15; 60G18

\section{Introduction}

This paper is devoted to the study of a multidimensional reflected fractional Brownian motion (RFBM) process on the positive orthant $S:=\mathbb{R}_{+}^{d}$, with drift $r^{0} \in \mathbb{R}^{d}$ and Hurst parameter $H \in\left(\frac{1}{2}, 1\right)$. Recently, Delgado [5] showed that the workload process of multistation fluid queueing network models with feedback and a nondeterministic arrival process, generated by a large number of heavy-tailed ON/OFF sources, can be approximated under suitable heavy traffic conditions by a multidimensional RFBM with Hurst parameter $H \in\left(\frac{1}{2}, 1\right)$. This model was further studied in subsequent papers [6], [7]. Besides [5], the multidimensional RFBM process has also been obtained as an approximating model in [15], in which a single-class queueing network with long-range dependent arrival and service processes was considered. It was shown therein that the normalized queue length process converges to a $d$-dimensional RFBM, with $d$ being the number of nodes or servers. Long time asymptotics and stability analysis of such a class of models are of fundamental interest. However, since an FBM is neither a semimartingale nor a Markov process, many techniques from the classical theory of stochastic calculus are inapplicable to its analysis.

In this work we establish a uniform moment estimate on expected return times of the RFBM process $\{Z(t): t \geq 0\}$ to a compact set; that is, we show that, for some $\delta, \kappa \in(0, \infty)$,

$$
\sup _{x \in B} \mathrm{E}_{x}\left[\tau_{B}(\delta)\right]<\infty,
$$

where $B=\{x \in S:|x| \leq \kappa\}, \tau_{B}(\delta)=\inf \{t \geq \delta: Z(t) \in B\}$, and $\mathrm{E}_{x}$ denotes the expectation conditional on the process $Z$ starting from $x \in S$. This result is reminiscent of a necessary

Received 6 July 2010; revision received 12 October 2010.

* Postal address: Department of Statistics, Colorado State University, Fort Collins, CO 80523, USA.

Email address: chihoon@stat.colostate.edu 
and sufficient condition for the positive Harris recurrence of Harris recurrent Markov processes (see, e.g. [16, Theorem 4.4], [17, Theorem 4.1] and [19, Chapter 11]). More precisely, for a wide class of Markov processes, condition (1.1), combined with a petite set requirement for $B$, implies the positive Harris recurrence of the process. Similar results as in (1.1) are known for reflected processes driven by standard Brownian motions; indeed, the positive Harris recurrence result of [11] (see also [14]), together with Theorem 4.4 of [16], implies (1.1) for semimartingale reflecting Brownian motions. We also refer the reader to Theorem 4.7 and Corollary 4.14 of [3] for more refined results. In this regard, results in this paper can be viewed as a significant step towards the further analysis of RFBM with the aim of establishing similar recurrent properties for reflected processes driven by non-Markovian processes.

The organization of the paper is as follows. In Section 2 we carefully describe our model in Definition 2.1 and make a standard assumption on the reflection matrix (see assumption (HR) in Section 2), which is used for invoking a functional central limit theorem in the heavy traffic analysis of [5] and [6]. In addition, similar to [14], we assume a natural stability condition (see condition (S) in Section 2) on the RFBM process. Our proof is based on uniform stability estimates (see the proof of Theorem 3.1, in particular (3.4)) on a family of certain deterministic dynamical systems obtained from a fluid limit analysis of the underlying RFBM process. This result, together with a maximal inequality for FBM with $H \in\left(\frac{1}{2}, 1\right)$, leads to uniform time estimates (Theorem 3.1) on the $p$ th $(p \geq 1)$ moment of the process in terms of its initial condition. In order to connect this result with moments of return times to a compact set, we deduce a drift inequality (see (3.11)), leading to the control of the expected overall hitting time, and establish the main result in Theorem 3.2.

We use the following notation. The set of positive integers is denoted by $\mathbb{N}$, the set of real numbers by $\mathbb{R}$, and the set of nonnegative real numbers by $\mathbb{R}_{+}$. Let $\mathbb{R}^{d}$ be the $d$-dimensional Euclidean space and, for $x \in \mathbb{R}^{d}$, the $L_{1}$ norm of $x$, i.e. $\sum_{i=1}^{d}\left|x_{i}\right|$, will be denoted by $|x|$. Let $\mathbb{R}^{d \times m}$ be the space of real $(d \times m)$-matrices with the norm $\|A\|=\max _{1 \leq j \leq m} \sum_{i=1}^{d}\left|a_{i j}\right|$ for $A \in \mathbb{R}^{d \times m}$. For a given matrix $M$, denote by $M^{\top}$ its transpose and by $M_{i}$ the $i$ th row of $M$. Let $\mathbb{I}=\mathbb{I}_{d \times d}$ denote the identity matrix for some $d$. When it is clear from the context, we will omit the subscript. For a set $A \subseteq \mathbb{R}^{d}$, denote its interior and boundary by $A^{\circ}$ and $\partial A$, respectively. For sets $A, B \subseteq \mathbb{R}^{d}, \operatorname{dist}(A, B)$ will denote the distance between two sets, i.e. $\inf \{|x-y|: x \in A, y \in B\}$. Let $C(X, Y)$ denote the space of continuous functions from $X$ to $Y$, endowed with the topology of uniform convergence on compact intervals. Inequalities for vectors are interpreted componentwise.

\section{Model and assumptions}

We begin with the definitions of multidimensional FBM and RFBM. Let $d \in \mathbb{N}$. A stochastic process $B_{H}=\left\{B_{H}(t)=\left(B_{H}^{(1)}(t), \ldots, B_{H}^{(d)}(t)\right)^{\top}, t \geq 0\right\}$, defined on some filtered probability space $\left(\Omega, \mathcal{F},\left(\mathcal{F}_{t}\right)_{t \geq 0}, \mathrm{P}\right)$, is called a $d$-dimensional FBM of (Hurst) parameter $H \in(0,1)$, starting from $B_{H}(0) \in \mathbb{R}^{d}$, with associated matrix $\Lambda$, if it is a continuous Gaussian process with initial condition $B_{H}(0), \mathrm{P}$-almost surely (P-a.s.) and with covariance function given by

$$
\operatorname{cov}\left(B_{H}(t), B_{H}(s)\right)=\mathrm{E}\left[\left(B_{H}(t)-B_{H}(0)\right)\left(B_{H}(s)-B_{H}(0)\right)^{\top}\right]=\Lambda_{H}(s, t) \Lambda
$$

for any $s, t \geq 0$, where $\Lambda$ is a $d \times d$ positive definite matrix and

$$
\Lambda_{H}(s, t)=\frac{1}{2}\left(t^{2 H}+s^{2 H}-|t-s|^{2 H}\right) .
$$

Also, it is assumed that $B_{H}$ is adapted to the filtration $\left(\mathcal{F}_{t}\right)_{t \geq 0}$. We will say that $B_{H}$ is a $d$-dimensional FBM with associated data $\left(B_{H}(0), H, \Lambda\right)$. 
Fix the column vectors $r^{0}, r^{1}, \ldots, r^{d} \in \mathbb{R}^{d}$, and let $R:=\left[r^{1}, \ldots, r^{d}\right]_{d \times d}$. We call the quintuple $\left(B_{H}(0), H, \Lambda, r^{0}, R\right)$ the data for an RFBM. The following definition is similar to that of [5].

Definition 2.1. (RFBM.) For $x \in S$, an RFBM associated with the data $\left(x, H, \Lambda, r^{0}, R\right)$ is a continuous $d$-dimensional process $Z$, defined on some probability space $(\Omega, \mathcal{F}, \mathrm{P})$, such that

(i) $Z(t)=x+B_{H}(t)+r^{0} t+R Y(t) \in S$ for all $t \geq 0$, P-a.s.,

(ii) $B_{H}$ is a $d$-dimensional FBM with data $(0, H, \Lambda)$,

(iii) $Y$ is a $d$-dimensional process such that $Y_{i}(0)=0$ for $i=1, \ldots, d$, P-a.s. For each $i=1, \ldots, d, Y_{i}$ is continuous, nondecreasing, and can increase only when $Z(\cdot)$ is on the face $F^{i}:=\left\{x \in S: x_{i}=0\right\}$, i.e. $\int_{0}^{t} \mathbf{1}_{\left\{Z_{i}(s) \neq 0\right\}} \mathrm{d} Y_{i}(s)=0$ for all $t \geq 0$.

For $y \in \partial S$, the set of directions of reflections is defined as

$$
r(y):=\left\{\sum_{i=1}^{d} q_{i} r^{i}: \sum_{i=1}^{d} q_{i}=1, q_{i} \geq 0, \text { and } q_{i}>0 \text { only if } y_{i}=0\right\} .
$$

To get an idea of the RFBM introduced in the above definition, we note that it behaves like an FBM in the interior of the orthant $S$ and is confined to the orthant by instantaneous 'reflection' at the boundary $\partial S$. For each $i$, the $i$ th column of the reflection matrix $R$ gives the direction of the reflection on the $i$ th face $F^{i}$. Specifically, if the boundary $F^{i}$ is hit, it is $Y_{i}$ that increases, the direction of displacement is given by $r^{i}$, the $i$ th column of $R$, and the magnitude of the displacement is the minimal amount required to keep $Z_{i}$ nonnegative. We refer the reader to [22] and the references therein for the related definition and properties of semimartingale reflecting Brownian motions in an orthant.

Remark 2.1. We call a square matrix $R$ completely- $\&$ if, for every $k \times k$ principal submatrix $G$ of $R$, there is a $k$-dimensional vector $v_{G}$ such that $v_{G} \geq 0$ and $G v_{G}>0$. The completely- $s$ condition on the reflection matrix $R$ ensures that, for every $x \in \partial S$, there exists a convex combination of vectors in $r(x)$ which points into $S^{\circ}$ from $x$. Also, the completely- $\&$ property is sufficient to ensure the existence of a pair $(Z, Y)$ satisfying Definition 2.1(i) and (iii) (cf. Theorem 2 of [2]). However, this property does not ensure the adaptness of the process $Y$ to a filtration to which $B_{H}$ is adapted. This problem is overcome under a stronger assumption on $R$, which we state below (cf. Proposition 4.2 of [22] and Section 2 of [5]).

Throughout this paper, we will impose the following assumption on the matrix $R$.

(HR) (i) The reflection matrix $R$ can be expressed as $\mathbb{I}+\Theta$, where $\Theta$ is a $d \times d$ matrix such that $|\Theta|$, that is, the matrix obtained from $\Theta$ by replacing all the entries in $\Theta$ by their absolute values, has spectral radius $r(|\Theta|)$ strictly less than 1 .

(ii) Moreover, the matrix $\Theta=\left(\theta_{i j}\right)$ satisfies $\theta_{i j} \leq 0$ and $\theta_{i i}=0$ for $1 \leq i, j \leq d$.

Part (i) of (HR) implies that $R$ is a completely- $\&$ matrix. Also, it can be shown (cf. [5] and [22]) that if $B_{H}$ is adapted to some filtration $\left\{\mathcal{F}_{t}: t \geq 0\right\}$ then $(Z, Y)$ is adapted to the filtration $\left\{g_{t}: t \geq 0\right\}$, with $g_{t}=\mathcal{F}_{t} \vee \mathcal{N}$, where $\mathcal{N}$ denotes the collection of P-null sets in $\mathcal{F}$. Furthermore, part (i) of (HR) is a sufficient condition for strong pathwise uniqueness of a solution of the Skorokhod problem described below. Henceforth, with an abuse of notation, we will assume that $(Z, Y)$ is adapted to the filtration $\left\{\mathcal{F}_{t}: t \geq 0\right\}$. 
We also note that the spectral radius of $-\Theta$ in $(\mathrm{HR})$ is strictly less than 1 , since $r(-\Theta)=$ $r(\Theta) \leq r(|\Theta|)$, where the inequality follows by Gelfand's theorem on the spectral radius [12] (that is, the spectral radius of a square matrix $A$ is given by $r(A)=\lim _{k \rightarrow \infty}\left\|A^{k}\right\|^{1 / k}$ with consistent matrix norm $\|\cdot\|$ on the space of matrices). Hence, $\mathbb{I}+(-\Theta)+(-\Theta)^{2}+\cdots$ is convergent and, therefore, part (i) of (HR) implies the existence of $R^{-1}$. Part (ii) of (HR), together with part (i), verifies the so-called Harrison-Reiman condition in [13]. Therefore, assumption (HR) will imply that a solution to the Skorokhod problem exists, and, moreover, the Skorokhod map is Lipschitz continuous in the sense of Proposition 2.1 below. (See also [9, pp. 163-165] and [10, pp. 200-208] for the generalized Harrison-Reiman condition for Lipschitz continuity of the Skorokhod map.)

Definition 2.2. (Skorokhod problem.) Let $\psi \in C\left([0, \infty), \mathbb{R}^{d}\right)$ be given with $\psi(0) \in S$. Then $(\phi, \eta) \in C\left([0, \infty), \mathbb{R}^{d}\right) \times C\left([0, \infty), \mathbb{R}^{d}\right)$ solves the Skorokhod problem for $\psi$ with respect to $S$ and $R$ if and only if the following assertions hold:

(i) $\phi(t)=\psi(t)+R \eta(t) \in S$ for all $t \geq 0$,

(ii) $\eta$ satisfies, for $1 \leq i \leq d$, (a) $\eta_{i}(0)=0$, (b) $\eta_{i}$ is nondecreasing, and (c) $\eta_{i}$ can increase only when $\phi$ is on the $i$ th face of $S$, that is, $\int_{0}^{\infty} \mathbf{1}_{\left\{\phi_{i}(s) \neq 0\right\}} \mathrm{d} \eta_{i}(s)=0$.

Let

$$
C_{S}\left([0, \infty), \mathbb{R}^{d}\right):=\left\{\psi \in C\left([0, \infty), \mathbb{R}^{d}\right): \psi(0) \in S\right\} .
$$

On the domain $D \subset C_{S}\left([0, \infty), \mathbb{R}^{d}\right)$, on which there is a unique solution to the Skorokhod problem, we define the Skorokhod map $\Gamma$ as

$$
\Gamma(\psi):=\phi
$$

if $\left(\phi, R^{-1}[\phi-\psi]\right)$ is the unique solution of the Skorokhod problem posed by $\psi$. An equivalent form of the RFBM in Definition 2.1 in terms of the Skorokhod map can now be written:

$$
Z=\Gamma\left(x+B_{H}+r^{0} \imath\right), \quad Z-\left(x+B_{H}+r^{0} \imath\right)=R Y .
$$

Here $\imath:[0, \infty) \rightarrow[0, \infty)$ is the identity map.

The following proposition gives the regularity of the Skorokhod map, which is a consequence of assumption (HR). We refer the reader to the proof of Equation (10) of [13, p. 305] and the arguments in [9, pp. 164-165] for its proof; although the Lipschitz continuity is not stated explicitly in [13], it follows easily from the method used to prove the existence of solutions and the continuity of the Skorokhod map (map $\phi(\cdot)$ in [13]). See also [8], [9], and [10] for more sufficient conditions under which this regularity property holds.

Proposition 2.1. The Skorokhod map is well defined on all of $C_{S}\left([0, \infty), \mathbb{R}^{d}\right)$, i.e. $D=$ $C_{S}\left([0, \infty), \mathbb{R}^{d}\right)$, and the Skorokhod map is Lipschitz continuous in the following sense. There exists a constant $L \in(1, \infty)$ such that, for all $\psi_{1}, \psi_{2} \in C_{S}\left([0, \infty), \mathbb{R}^{d}\right)$ and $t \geq 0$,

$$
\sup _{0 \leq s \leq t}\left|\Gamma\left(\psi_{1}\right)(s)-\Gamma\left(\psi_{2}\right)(s)\right|<L \sup _{0 \leq s \leq t}\left|\psi_{1}(s)-\psi_{2}(s)\right| .
$$

Finally, we introduce the following condition on the drift vector $r^{0}$ and the matrix $R$ that will be assumed throughout this paper.

(S) There exists a $\theta>0$ such that $\sup _{1 \leq i \leq d}\left[R^{-1} r^{0}\right]_{i}<-\theta$. 
Remark 2.2. For a model driven by regular Brownian motion (i.e. $H=\frac{1}{2}$ ), condition (S) is known as a necessary and sufficient condition for the existence of a unique stationary distribution. See [14] and the references therein for the related asymptotic stability conditions of reflected Brownian motions on a positive orthant.

\section{Main results}

Let $Z_{x}$ be defined as in Definition 2.1, with $Z_{x}(0)=x \in S$ and Hurst parameter $H \in\left(\frac{1}{2}, 1\right)$. In what follows, note that $\mathrm{E}\left|Z_{x}(t)\right|$ can be alternatively written as $\mathrm{E}_{x}|Z(t)|$. The following moment stability properties are key ingredients in the proofs.

Theorem 3.1. There exists a $\delta \in(0, \infty)$ such that, for all $p \geq 1$,

$$
\lim _{|x| \rightarrow \infty} \frac{1}{|x|^{p}} \mathrm{E}\left[\left|Z_{x}(\delta|x|)\right|^{p}\right]=0 .
$$

Proof. Fix $x \in S$ and $p \geq 1$. We write the RFBM $Z$ in Definition 2.1 as

$$
Z_{x}(t)=\Gamma\left(x+r^{0} l+B_{H}(\cdot)\right)(t), \quad t \geq 0,
$$

where $\imath:[0, \infty) \rightarrow[0, \infty)$ is the identity map. Define the deterministic trajectory

$$
z_{x}(t)=\Gamma\left(x+r^{0} l\right)(t), \quad t \geq 0 .
$$

Using the Lipschitz property of $\Gamma$ (Proposition 2.1), we have

$$
\left|Z_{x}(t)-z_{x}(t)\right| \leq L \sup _{0 \leq s \leq t}\left|B_{H}(s)\right| \quad \text { for all } t \geq 0 .
$$

Next, defining $\mathcal{C}:=\left\{v \in \mathbb{R}^{d}: R^{-1} v \leq 0\right\}$ we see from condition (S) that there exists a $\beta \in(0, \infty)$ satisfying

$$
\begin{aligned}
\operatorname{dist}\left(r^{0}, \partial \mathcal{C}\right) & =\inf \left\{\left|r^{0}-v\right|: R^{-1} v=0, v \in \mathbb{R}^{d}\right\} \\
& \geq \frac{1}{\left\|R^{-1}\right\|} \inf \left\{\left|R^{-1} r^{0}-R^{-1} v\right|: R^{-1} v=0, v \in \mathbb{R}^{d}\right\} \\
& =\frac{1}{\left\|R^{-1}\right\|}\left|R^{-1} r^{0}\right| \\
& \geq \frac{1}{\left\|R^{-1}\right\|} \theta d \\
& =: \beta \\
& >0
\end{aligned}
$$

where $\theta \in(0, \infty)$ is a constant, as in condition (S), and the first inequality is due to the fact that $|A x| \leq \| A|||x|$ for a matrix $A_{d \times d}$ and a vector $x_{d \times 1}$. Thus,

$$
r^{0} \in \mathcal{C}_{\beta}:=\{v \in \mathcal{C}: \operatorname{dist}(v, \partial \mathcal{C}) \geq \beta\}
$$

For $x_{0} \in S$, denote by $\mathcal{K}\left(x_{0}\right)$ the collection of all trajectories $\psi:[0, \infty) \rightarrow S$ of the form

$$
\psi(t)=\Gamma\left(x_{0}+\varpi \iota\right)(t), \quad t \geq 0,
$$


where $\varpi$ ranges over all of $\mathcal{C}_{\beta}$. Define the 'hitting time to the origin' function as

$$
T\left(x_{0}\right):=\sup _{\psi \in \mathcal{K}\left(x_{0}\right)} \inf \{t \in[0, \infty): \psi(t)=0\} .
$$

Then, owing to Lemma 3.1 of [1] we have

$$
T\left(x_{0}\right) \leq \frac{4 L^{2}}{\beta}\left|x_{0}\right|, \quad \text { and, for all } \psi \in \mathcal{K}\left(x_{0}\right), \quad \psi(t)=0 \quad \text { for all } t \geq T\left(x_{0}\right) .
$$

Combining this observation with (3.3), we now have $z_{x}(t)=0$ for all $t \geq \delta_{0}|x|$, where $\delta_{0}:=4 L^{2} / \beta$. Using this in (3.2), we now see that

$$
\left|Z_{x}(t|x|)\right| \leq L \sup _{0 \leq s \leq t|x|}\left|B_{H}(s)\right|
$$

for all $t \geq \delta_{0}$ and all initial conditions $x$. Next we obtain an estimate on the $p$ th moment of the right-hand side of (3.5). For $H \in\left(\frac{1}{2}, 1\right)$, it is known from Theorem 1.2 of [20] (see also Example 5.1.5 of [21]) that

$$
\mathrm{E}\left(\sup _{0 \leq s \leq t}\left|B_{H}^{(i)}(s)\right|\right)^{p} \leq C(p, H) t^{p H},
$$

where $i=1, \ldots, d$ and $C(p, H) \in(0, \infty)$ is a constant which depends only on $p$ and $H$. Applying this estimate in (3.5), we now have, for all $t \geq \delta_{0}$ and $x \in S$,

$$
\mathrm{E}\left|Z_{x}(t|x|)\right|^{p} \leq C(t|x|)^{p H}
$$

for some constant $C \in(0, \infty)$, which depends only on $p$ and $H$. The result now follows on choosing any $\delta \geq \delta_{0}$ since $H<1$.

Remark 3.1. We note that (3.1) with $p=1$ was established in [4] for a wide class of Markov processes. Combined with a certain petite set requirement, it was shown in [4] that (3.1) with $p=1$ implies the positive Harris recurrence of underlying Markov processes.

We now present the main result on return times to a compact set for the RFBM process. Its proof follows along similar lines to the proofs presented in Theorem 3.1 of [4] and Theorem 2.1(ii) of [18].

Theorem 3.2. Let $B=\{x \in S:|x| \leq \kappa\}$ for some $\kappa \geq 1$. Then

$$
\sup _{x \in B} \mathrm{E}_{x}\left[\tau_{B}(\delta)\right]<\infty
$$

where $\tau_{B}(\delta):=\inf \{t \geq \delta: Z(t) \in B\}$ with some $\delta>0$.

Proof. By Theorem 3.1 (with $p=1$ ), there exists a $\kappa \geq 1$ such that, with $B=\{x \in$ $S:|x| \leq \kappa\}$

$$
\mathrm{E}\left|Z_{x}(\delta|x|)\right| \leq \frac{1}{2}|x| \text { for all } x \in B^{\mathrm{c}}
$$

where $\delta$ is as in Theorem 3.1. Analogously to (3.6), we have $\mathrm{E}\left|Z_{x}(t)\right|^{p} \leq C t^{p H}$ for any $t \geq \delta_{0}|x|$ and some constant $C>0$. Take $\delta=\delta_{0} \kappa\left(\geq \delta_{0}\right)$. Then, with $p=1$ and $t=\delta$ $\left(\geq \delta_{0}|x|\right.$ for $x \in B$ ), we have, for some constant $b>0$,

$$
\mathrm{E}\left|Z_{x}(\delta)\right| \leq b \quad \text { for all } x \in B .
$$


For $x \in S$, let

$$
n(x)= \begin{cases}\delta|x| & \text { if } x \in B^{\mathrm{c}} \\ \delta & \text { if } x \in B\end{cases}
$$

Since $\kappa \geq 1, n(x) \geq \delta$ for all $x \in S$. It follows from (3.8) and (3.9) that

$$
\mathrm{E}\left|Z_{x}(n(x))\right| \leq \frac{1}{2}|x|+b \mathbf{1}_{B}(x) \leq|x|-\frac{1}{2 \delta} n(x)+\tilde{b} \mathbf{1}_{B}(x)
$$

for some $\tilde{b} \geq \frac{1}{2}+b>0$ and all $x \in S$.

In order to prove (3.7), we work with an 'embedded' process $\breve{Z}$. Note that $n(x)$ in (3.10) is a (trivial) stopping time. For $k \geq 1$, let $s(k)$ denote its iterates; that is, along any sample paths,

$$
s(0)=0, \quad s(1)=n(x), \quad \text { and } \quad s(k+1)=s(k)+n\left(Z_{x}(s(k))\right) .
$$

Define

$$
\breve{Z}(k):=Z_{x}(s(k)), \quad \breve{\mathscr{F}}_{k}:=\widetilde{F}_{s}(k), \quad \text { and } \quad \breve{\tau}_{B}(\delta):=\inf \{k \geq \delta: \breve{Z}(k) \in B\} .
$$

Then $s\left(\breve{\tau}_{B}(\delta)\right)$ denotes the time of the first return to $B$ by the original process along an embedded path; that is,

$$
s\left(\breve{\tau}_{B}(\delta)\right)=\sum_{k=0}^{\breve{\tau}_{B}(\delta)-1} n(\breve{Z}(k))
$$

and so we have, a.s.,

$$
s\left(\breve{\tau}_{B}(\delta)\right) \geq \tau_{B}(\delta)
$$

We claim that

$$
\mathrm{E}_{x}\left[\sum_{k=0}^{\breve{\tau}_{B}(\delta)-1} n(\breve{Z}(k))\right] \leq 2 \delta(|x|+\tilde{b}) .
$$

Then, we have, for each $x \in S$, by adding the lengths of the embedded time $n(x)$ along any sample path, and from (3.12)-(3.14),

$$
\mathrm{E}_{x}\left[\tau_{B}(\delta)\right] \leq \mathrm{E}_{x}\left[\sum_{k=0}^{\breve{\tau}_{B}(\delta)-1} n(\breve{Z}(k))\right] \leq 2 \delta(|x|+\tilde{b}) .
$$

Hence,

$$
\sup _{x \in B} \mathrm{E}_{x}\left[\tau_{B}(\delta)\right] \leq 2 \delta\left(\sup _{x \in B}|x|+\tilde{b}\right)=2 \delta(\kappa+\tilde{b})<\infty .
$$

Thus, it only remains to prove the claim in (3.14). For $n \geq 1$, define

$$
\tau^{n}:=\min \left\{n, \breve{\tau}_{B}(\delta), \inf \{k \geq 0: \breve{Z}(k) \geq n\}\right\} .
$$

Note that, since $\left\{\tau^{n} \geq i\right\} \in \breve{\mathcal{F}}_{i-1}$, we have

$$
\mathrm{E}_{x}\left[\breve{Z}\left(\tau^{n}\right)\right]=\mathrm{E}_{x}[\breve{Z}(0)]+\mathrm{E}_{x}\left[\sum_{i=1}^{\tau^{n}}\left(\mathrm{E}_{x}\left[\breve{Z}(i) \mid \breve{\mathcal{F}}_{i-1}\right]-\breve{Z}(i-1)\right)\right] .
$$


Fix $N>0$. From (3.11) and (3.15), we see that

$$
0 \leq \mathrm{E}_{x}\left[\breve{Z}\left(\tau^{n}\right)\right] \leq|x|+\mathrm{E}_{x}\left[\sum_{i=1}^{\tau^{n}}\left(\tilde{b} \mathbf{1}_{B}(\breve{Z}(i-1))-\frac{1}{2 \delta} n(\breve{Z}(i-1)) \wedge N\right)\right] .
$$

Hence, by adding a finite term to each side of (3.16), we get

$$
\begin{aligned}
\mathrm{E}_{x}\left[\sum_{i=1}^{\tau^{n}} \frac{1}{2 \delta} n(\breve{Z}(i-1)) \wedge N\right] & \leq|x|+\mathrm{E}_{x}\left[\sum_{i=1}^{\tau^{n}} \tilde{b} \mathbf{1}_{B}(\breve{Z}(i-1))\right] \\
& \leq|x|+\mathrm{E}_{x}\left[\sum_{i=1}^{\breve{\tau}_{B}(\delta)} \tilde{b} \mathbf{1}_{B}(\breve{Z}(i-1))\right] \\
& \leq|x|+\tilde{b} .
\end{aligned}
$$

Letting $n \rightarrow \infty$ and then $N \rightarrow \infty$ gives the result by the monotone convergence theorem.

\section{Acknowledgements}

The author would like to thank the associate editor and the anonymous referee for carefully examining the paper and providing a number of valuable comments that led to several important improvements.

\section{References}

[1] Atar, R., Budhiraja, A. And Dupuis, P. (2001). On positive recurrence of constrained diffusion processes. Ann. Prob. 29, 979-1000.

[2] Bernard, A. and el Kharroubi, A. (1991). Régulations déterministes et stochastiques dans le premier "orthant" de $\mathbf{R}^{n}$. Stoch. Stoch. Reports 34, 149-167.

[3] Budhiraja, A. AND LeE, C. (2007). Long time asymptotics for constrained diffusions in polyhedral domains. Stoch. Process. Appl. 117, 1014-1036.

[4] DAI, J. G. (1995). On positive Harris recurrence of multiclass queueing networks: a unified approach via fluid limit models. Ann. Appl. Prob. 5, 49-77.

[5] Delgado, R. (2007). A reflected fBm limit for fluid models with ON/OFF sources under heavy traffic. Stoch. Process. Appl. 117, 188-201.

[6] Delgado, R. (2008). State space collapse for asymptotically critical multi-class fluid networks. Queueing Systems 59, 157-184.

[7] Delgado, R. (2010). On the reflected fractional Brownian motion process on the positive orthant: asymptotics for a maximum with application to queueing networks. Stoch. Models 26, 272-294.

[8] Dupuis, P. AND IsHII, H. (1991). On Lipschitz continuity of the solution mapping to the Skorokhod problem, with applications. Stoch. Stoch. Reports 35, 31-62.

[9] Dupuis, P. and Ramanan, K. (1999). Convex duality and the Skorokhod problem. I. Prob. Theory Relat. Fields 115, 153-195.

[10] Dupuis, P. and Ramanan, K. (1999). Convex duality and the Skorokhod problem. II. Prob. Theory Relat. Fields 115, 197-236.

[11] Dupuis, P. AND Williams, R. J. (1994). Lyapunov functions for semimartingale reflecting Brownian motions. Ann. Prob. 22, 680-702.

[12] Gelfand, I. (1941). Normierte Ringe. Rec. Math. [Mat. Sbornik] N. S. 9, 3-24.

[13] Harrison, J. M. and Reiman, M. I. (1981). Reflected Brownian motion on an orthant. Ann. Prob. 9, 302-308.

[14] Harrison, J. M. AND Williams, R. J. (1987). Brownian models of open queueing networks with homogeneous customer populations. Stochastics 22, 77-115.

[15] Konstantopoulos, T. and Lin, S.-J. (1996). Fractional Brownian approximations of queueing networks. In Stochastic Networks (New York, 1995; Lecture Notes Statist. 117), Springer, New York, pp. 257-273.

[16] Meyn, S. P. ANd Tweedie, R. L. (1993). Stability of Markovian processes. II. Continous-time processes and sampled chains. Adv. Appl. Prob. 25, 497-517. 
[17] Meyn, S. P. And Tweedie, R. L. (1993). Stability of Markovian processes. III. Foster-Lyapunov criteria for continous-time processes. Adv. Appl. Prob. 25, 518-548.

[18] Meyn, S. P. And Tweedie, R. L. (1994). State-dependent criteria for convergence of Markov chains. Ann. Appl. Prob. 4, 149-168.

[19] Meyn, S. And Tweedie, R. L. (2009). Markov Chains and Stochastic Stability. Cambridge University Press.

[20] Novikov, A. and Valkeila, E. (1999). On some maximal inequalities for fractional Brownian motions. Statist. Prob. Lett. 44, 47-54.

[21] Nualart, D. (2006). The Malliavin Calculus and Related Topics, 2nd edn. Springer, Berlin.

[22] Williams, R. J. (1998). An invariance principle for semimartingale reflecting Brownian motions in an orthant. Queueing Systems 30, 5-25. 